\title{
Local Shape Transfer for Image Co-segmentation
}

Wei Teng ${ }^{1}$

tengw@buaa.edu.cn

Yu Zhang ${ }^{1}$

zhangyulb@gmail.com

Xiaowu Chen ${ }^{\dagger 1}$

chen@buaa.edu.cn

Jia Li' ${ }^{12}$

jiali@buaa.edu.cn

Zhiqiang $\mathrm{He}^{3}$

lirong2@lenovo.com
${ }^{1}$ State Key Laboratory of Virtual Reality

Technology and Systems

Beihang University

Beijing, China

${ }^{2}$ International Research Institute for Multidisciplinary Science

Beihang University

Beijing, China

${ }^{3}$ Lenovo Research
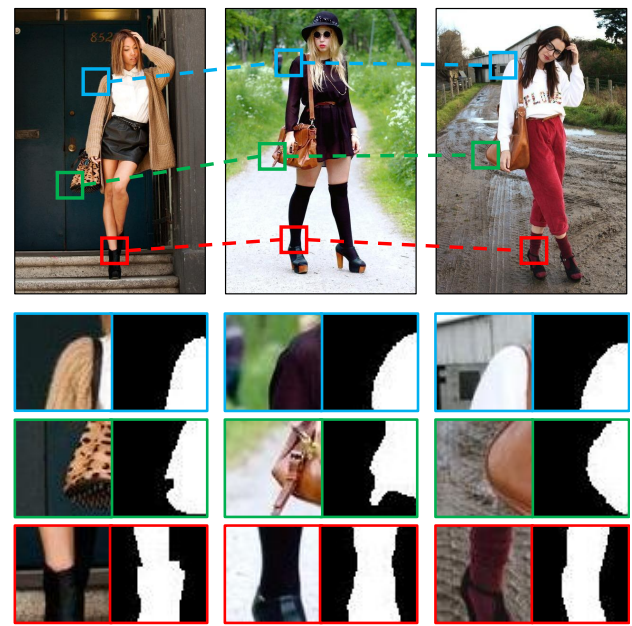

Figure 1: The motivation of this paper. The common objects in these images have different poses, rendering their global shapes inconsistent. However, the local object shapes in different images are highly consistent and provide important cues for co-segmentation.

Image co-segmentation is a challenging computer vision task that aims to segment all pixels of the common objects in an image set. In real-world cases, the common objects often vary greatly in poses, locations and scales, making their global shapes highly inconsistent across images and difficult to be segmented. However their local shapes are often highly consistent (see Fig. 1) and thus transferable. Based on the observation, we propose a novel co-segmentation approach, which transfers patch-level local object shapes and appears more consistently across different images. Given a group of $M$ images,

$\dagger$ correspondence should be addressed to Xiaowu Chen. our framework first estimates coarse initial foreground segmentations by thresholding saliency maps [3]. Meanwhile, we build inter-image connections by constructing a weighted graph on patches sampled from different images using [1], where weights are learned by Locally Linear Embedding [2]. With the patch graph, we refine the initial segmentation in each image by transferring the local shapes among different images. Formally, we minimize the objective

$$
\begin{gathered}
\min _{\mathbf{y}} \sum_{i=1}^{M} E_{\mathrm{seg}}\left(\mathbf{y}^{[i]}\right)+\alpha \sum_{i=1}^{P}\left\|\vec{y}_{i}-\sum_{j \in N_{i}} w_{i j} \vec{y}_{j}\right\|^{2}, \\
\text { s.t. } \mathbf{y} \in\{0,1\}|\mathbf{y}|
\end{gathered}
$$

where $\mathbf{y}$ concatenates the binary labels of all pixels in the image set, $\mathbf{y}^{[i]}$ is the part from the $i$ th image. The energy $E_{\text {seg }}$ implements intra-image foreground/background segmentation, for which we use the popular Markov Random Field energy. The problem is NP-hard and usually large scale as it operates on pixels, which is approximately solved by half quadratic splitting.

We evaluate the proposed approach on two public benchmarks: iCoseg and Fashionista. Experiments show that our approach performs comparably with or better than the state-of-the-arts on iCoseg dataset, while achieving more than $31 \%$ relative improvements on Fashionista dataset.

[1] J. Kim, C. Liu, F. Sha, and K. Grauman. Deformable spatial pyramid matching for fast dense correspondences. In CVPR, pages 2307-2314, 2013.

[2] S. Roweis and L. Saul. Nonlinear dimensionality reduction by locally linear embedding. Science, 290:2323-2326, 2000.

[3] J. Zhang, S. Sclaroff, Z. Lin, X. Shen, and B. Price. Minimum barrier salient object detection at $80 \mathrm{fps}$. In ICCV , pages 1404-1412, 2015. 